\title{
Comparison of Diffusion-Weighted MRI Reconstruction Methods for Visualization of Cranial Nerves in Posterior Fossa Surgery
}

\author{
Brendan Behan ${ }^{1,2}$, David Q. Chen ${ }^{1,3}$, Francesco Sammartino ${ }^{2}$, Danielle D. DeSouza ${ }^{1,4}$, \\ Erika Wharton-Shukster ${ }^{1,2}$ and Mojgan Hodaie ${ }^{1,2,3,5 *}$ \\ ${ }^{1}$ Division of Brain, Imaging and Behaviour - Systems Neuroscience, Krembil Institute, University Health Network, Toronto, \\ ON, Canada, ${ }^{2}$ Division of Neurosurgery, Department of Surgery, Toronto Western Hospital, Toronto, ON, Canada, \\ ${ }^{3}$ Department of Surgery, Institute of Medical Science, University of Toronto, Toronto, ON, Canada, ${ }^{4}$ Department of Neurology \\ and Neurological Sciences, Stanford University, Stanford, CA, United States, ${ }^{5}$ Joint Department of Medical Imaging, \\ University Health Network, Toronto, ON, Canada
}

\section{OPEN ACCESS}

Edited by:

Maxime Descoteaux,

Université de Sherbrooke, Canada

Reviewed by:

Arnaud Bore,

Centre de Recherche de l'Institut Universitaire de Gériatrie de Montréal,

Canada

Chao Yi-Ping

Chang Gung University, Taiwan

*Correspondence:

Mojgan Hodaie

mojgan.hodaie@uhn.ca

Specialty section:

This article was submitted to Brain Imaging Methods,

a section of the journal

Frontiers in Neuroscience

Received: 01 April 2017 Accepted: 21 September 2017 Published: 09 October 2017

Citation:

Behan B, Chen DQ, Sammartino F, DeSouza DD, Wharton-Shukster E and Hodaie M (2017) Comparison of

Diffusion-Weighted MRI

Reconstruction Methods for Visualization of Cranial Nerves in

Posterior Fossa Surgery.

Front. Neurosci. 11:554.

doi: 10.3389/fnins.2017.00554
Diffusion-weighted imaging (DWI)-based tractography has gained increasing popularity as a method for detailed visualization of white matter (WM) tracts. Different imaging techniques, and more novel, advanced imaging methods provide significant WM structural detail. While there has been greater focus on improving tract visualization for larger WM pathways, the relative value of each method for cranial nerve reconstruction and how this methodology can assist surgical decision-making is still understudied. Images from 10 patients with posterior fossa tumors (4 male, mean age: 63.5), affecting either the trigeminal nerve (CN V) or the facial/vestibular complex (CN VII/VIII), were employed. Three distinct reconstruction methods [two tensor-based methods: single diffusion tensor tractography (SDT) (3D Slicer), eXtended streamline tractography (XST), and one fiber orientation distribution (FOD)-based method: streamline tractography using constrained spherical deconvolution (CSD)-derived estimates (MRtrix3)], were compared to determine which of these was best suited for use in a neurosurgical setting in terms of processing speed, anatomical accuracy, and accurate depiction of the relationship between the tumor and affected $\mathrm{CN}$. Computation of the tensor map was faster when compared to the implementation of CSD to provide estimates of FOD. Both XST and CSD-based reconstruction methods tended to give more detailed representations of the projections of CN V and CN VII/VIII compared to SDT. These reconstruction methods were able to more accurately delineate the course of $\mathrm{CN} V$ and $\mathrm{CN}$ VII/VIII, differentiate $\mathrm{CN} V$ from the cerebellar peduncle, and delineate compression of CN VII/VIII in situations where SDT could not. However, CSD-based reconstruction methods tended to generate more invalid streamlines. XST offers the best combination of anatomical accuracy and speed of reconstruction of cranial nerves within this patient population. Given the possible anatomical limitations of single tensor models, supplementation with more advanced tensor-based reconstruction methods might be beneficial.

Keywords: diffusion tensor imaging, extended streamline tractography, constrained spherical deconvolution, fiber orientation distribution, trigeminal nerve, vestibulocochlear nerve 


\section{INTRODUCTION}

Diffusion-weighted imaging (DWI) is a neuroimaging method that assays the random movement of water molecules to reconstruct the structure of white matter (WM) fibers (Behrens and Johansen-Berg, 2009; Jones et al., 2013; Soares et al., 2013). Within the brain, this movement is affected by structural features such as axons of WM (Mori and van Zijl, 2002; O'Donnell and Westin, 2011). Consequently, the projections of WM tracts can be reconstructed based on their diffusion profiles-a technique termed "fiber tracking" or "tractography" (Conturo et al., 1999; Mori and Barker, 1999; Basser et al., 2000).

Providing detailed information about WM tracts in vivo makes tractography an attractive option for neurosurgical practice (Abdullah et al., 2013). Deterministic single diffusion tensor tractography (SDT) has been successfully used to reconstruct representations of large WM fiber tracts within patient populations with supratentorial tumors (Potgieser et al., 2014). While this method tends to provide reliable results when there is one major fiber bundle of interest, it performs less so in areas where multiple WM fiber bundles are present (Wedeen et al., 2008). This is due to its inherent limitation that it assumes each WM voxel has a single fiber orientation (Basser et al., 1994; O'Donnell and Westin, 2011). With an estimated 63-90\% of WM voxels containing multiple fiber bundles (Jeurissen et al., 2013), this can be a considerable problem for neurosurgical planning, particularly in terms of following the anatomical course of a nerve when it encounters other fiber bundles.

Given these limitations, attention has turned to developing other methods that satisfactorily deal with the occurrence of multiple fibers in a WM voxel. This can broadly be defined as high angular resolution diffusion imaging (HARDI) which requires more advanced DWI acquisition protocols (Tuch et al., 2002). One option is to glean more information from the original tensor map for fiber reconstruction purposes. EXtended Streamline Tractography (XST) is one such method that is based on twotensor reconstruction allowing for crossing fiber pathways to be reconstructed (Qazi et al., 2009). XST has been demonstrated to be superior to SDT in its generation of lateral projections of the corticospinal tract, but its efficacy has not been determined in smaller WM tracts.

Another option is to employ methods other than tensorbased approaches to characterize diffusion profiles (Tournier et al., 2011). An emerging popular method involves a nonnegativity constrained spherical deconvolution (CSD)-derived estimation of the distribution of fiber bundles per WM voxela fiber orientation distribution (FOD) estimate (Tournier et al., 2004, 2007). Similar to XST, this reconstruction method has been demonstrated to illustrate more accurate anatomical depictions of the corticospinal tract (Farquharson et al., 2013) and cerebellocortical tracts (Palesi et al., 2015), compared to conventional diffusion tensor reconstruction methods. However there has not been as much focus on its application in smaller WM tracts.

SDT has previously been successfully used to image cranial nerves (CNs) in both healthy controls and patients with tumors in the posterior cranial fossa (Kabasawa et al., 2007; Hodaie et al., 2010, 2012; Chen et al., 2011; Gerganov et al., 2011; Roundy et al., 2012; Yoshino et al., 2015; Hilly et al., 2016). Considering that $\mathrm{CN}$ projections often pass through regions of multiple fiber populations, and SDT's inability to resolve such arrangements, it is important to evaluate other reconstruction methods that may offer better depictions. Both HARDI-based reconstruction methods, XST and CSD-based streamline tractography, have produced more accurate depictions of larger WM tracts, compared to SDT. As such, we are interested to see whether we would see similar improvements in the visualization of smaller fiber bundles, such as CNs. Here, we aim to compare and contrast the aforementioned three discrete reconstruction methods with regards to the generation of the WM bundles of the trigeminal nerve (CN V), the facial nerve (CN VII), and the vestibulocochlear nerve (CN VIII). CN VII and CN VIII will be hereafter grouped together as one entity-the facial/vestibular complex (CN VII/VIII) - as both fibers tend to be closely aligned with one another in diffusion-weighted scans (Hodaie et al., 2010).

We use a cohort of patients with unilateral posterior fossa tumors to determine which of these aforementioned reconstruction methods provides optimal visualization of the $\mathrm{CN}$ fibers. Assessment is made based on the important practical criteria necessary for neurosurgical implementation, including processing speed, anatomical accuracy of fiber representations, and most importantly, the depiction of the relationship between $\mathrm{CN}$ fiber bundles and the tumor in the posterior cranial fossa. Furthermore, we use these criteria to determine whether HARDIbased methods, that do not assume one fiber orientation per voxel, show improved visualization parameters compared with single tensor-based models.

\section{MATERIALS AND METHODS \\ Patient Demographics}

Ten patients (4 male and 6 female, mean age: 63.5 , range: $48-85$ ), with cerebellopontine angle tumors, undergoing Gamma Knife radiosurgery treatment, were recruited for this study (for clinical demographic information, see Table 1). Four patients presented with tumors primarily affecting the trigeminal nerve (three meningiomas and one trigeminal schwannoma: P01-P04), with the remaining six presenting with vestibular schwannomas (P05P10). Institutional Review Board approval was obtained. Patients had not received prior surgical or radiosurgery treatment.

\section{Imaging}

Magnetic resonance images were acquired using GE Signa HDx 3T scanner with an 8 channel head-coil. MR sequences were acquired from a group of patients with tumors in the posterior cranial fossa. Diffusion weighted images (DWI) were acquired with $1 \mathrm{~B} 0$ scan, 60 gradient directions, $3 \mathrm{~mm}$ slice thickness and in-plane resolution of $0.9375 \times 0.9375 \mathrm{~mm}, \mathrm{~b} 0=1,000 \mathrm{~s} / \mathrm{mm}^{2}$, $\mathrm{TE}=88.6 \mathrm{~ms}, \mathrm{TR}=17,000 \mathrm{~ms}$, flip angle $=90^{\circ}$, matrix $=$ $128 \times 128$, number of slices $=44$. The scan acquisition time was $\sim 17.5$ minutes (min). T1 FSPGR anatomical scans were acquired with $1 \mathrm{~mm}$ slice thickness and in-plane resolution of $0.3906 \times 0.3906 \mathrm{~mm}$, slice spacing $=1 \mathrm{~mm}, \mathrm{TE}=3.72 \mathrm{~ms}$, TR $=9.06 \mathrm{~ms}$, flip angle $=12^{\circ}$, matrix $=320 \times 320$. T1 fast image 
TABLE 1 | Patient details with type of posterior cranial fossa tumor and properties.

\begin{tabular}{|c|c|c|c|c|c|}
\hline Patient & Age & Tumor type & $\begin{array}{l}\text { Volume } \\
\left(\mathrm{mm}^{3}\right)\end{array}$ & $\begin{array}{c}\text { Maximum } \\
\text { dimension }(\mathrm{mm})\end{array}$ & Architecture \\
\hline 1 & 54 & $\begin{array}{l}\text { Petrous } \\
\text { Meningioma }\end{array}$ & $2,201.41$ & 19.1 & Solid \\
\hline 2 & 48 & $\begin{array}{l}\text { Trigeminal } \\
\text { Schwannoma }\end{array}$ & 336.66 & 12.3 & Solid \\
\hline 3 & 75 & $\begin{array}{l}\text { Petroclival } \\
\text { Meningioma }\end{array}$ & $1,305.48$ & 18.5 & Solid \\
\hline 4 & 54 & Meningioma & 983.47 & 18.9 & Solid \\
\hline 5 & 73 & $\begin{array}{l}\text { Vestibular } \\
\text { Schwannoma }\end{array}$ & $1,246.07$ & 21.1 & Solid \\
\hline 6 & 71 & $\begin{array}{l}\text { Vestibular } \\
\text { Schwannoma }\end{array}$ & $8,409.41$ & 28.0 & Cystic \\
\hline 7 & 50 & $\begin{array}{l}\text { Vestibular } \\
\text { Schwannoma }\end{array}$ & 8,020 & 29.0 & Solid \\
\hline 8 & 71 & $\begin{array}{l}\text { Vestibular } \\
\text { Schwannoma }\end{array}$ & 355.86 & 11.5 & Solid \\
\hline 9 & 54 & $\begin{array}{l}\text { Vestibular } \\
\text { Schwannoma }\end{array}$ & $1,266.09$ & 20.0 & Solid \\
\hline 10 & 85 & $\begin{array}{l}\text { Vestibular } \\
\text { Schwannoma }\end{array}$ & $2,629.72$ & 23.3 & Cystic \\
\hline
\end{tabular}

employing steady state acquisition (FIESTA) scans were acquired with $0.8 \mathrm{~mm}$ slice thickness and in-plane resolution of $0.352 \times$ $0.352 \mathrm{~mm}$, slice spacing $=0.4 \mathrm{~mm}, \mathrm{TE}=2.4 \mathrm{~ms}, \mathrm{TR}=4.94 \mathrm{~ms}$, flip angle $=37^{\circ}$, matrix $=256 \times 256$.

\section{DWI Processing}

DWI sequences were initially corrected for eddy-current and motion distortions, through an affine transformation, using FLIRT (FMRIB's linear registration tool) in FSL (version 5.0.8) (Jenkinson et al., 2002, 2012). Custom MATLAB scripts were used to correct gradients for motion effects.

\section{Seed Placement}

Seeds for CN V were initially placed on retrogasserian portions of the nerve, as this was the most identifiable portion of the nerve and a common target for radiosurgical treatments (Regis et al., 2002; Massager et al., 2004). Seeds for CN VII/VIII were initially placed in the intracanalicular portion, as this was the most identifiable portion, particularly in patients presenting with vestibular schwannomas (P05-P10). Seeds for both $\mathrm{CN}$ V and $\mathrm{CN}$ VII/VIII were placed bilaterally in all patients. All seeds were mapped so as to incorporate a cross section of the $\mathrm{CN}$ of interest and were not more than 20 voxels in size. The same seeding region was used for all three reconstruction methods under consideration.

\section{Tensor-Based Methods}

\section{Single Diffusion Tensor Tractography (SDT)}

Images were imported into 3D Slicer version 3.6 (NA-MIC ${ }^{\complement}$, http://www.slicer.org) (Fedorov et al., 2012) on a Ubuntu 12.04 LTS OS for SDT, where a tensor map was created from the DWI scan using a least-squares method-the "DWI to DTI Estimation" module in 3D Slicer. Tractography parameters were initiated with seed spacing $=0.3 \mathrm{~mm}$, seeding FA threshold $=0.15$, stopping $\mathrm{FA}$ value $=0.15$, curvature threshold $=$ 0.8 , minimal length $=5 \mathrm{~mm}$, and integration distance $=0.1$. Tractography was performed with the "Labelmap Seeding" module in the 3D Slicer graphical user interface (GUI). Graphical representations of this reconstruction method are detailed elsewhere (Mori et al., 1999).

\section{EXtended Streamline Tractography (XST)}

Images were analyzed with XST software (Qazi et al., 2009). The tensor map created for SDT was also used for XST. Tractography parameters were initiated with seed spacing $=$ $0.3 \mathrm{~mm}$, stopping $\mathrm{FA}$ value $=0.15$, stopping linear anisotropy (as measured by $\mathrm{C}_{1}$ - Westin et al., 2002) $=0.1$, stopping fraction of the chosen tensor component $=0.1$, minimal length $=5 \mathrm{~mm}$, and curvature threshold $=0.8$. This method was implemented by using the "ten2fiber" command from a modified version of TEEM toolkit (https://github.com/sinkpoint/hodaieteem). Graphical representations of this reconstruction method are detailed elsewhere (Qazi et al., 2009).

\section{Fiber Orientation Distribution(FOD)-Based Method Constrained Spherical Deconvolution (CSD)}

Images were analyzed with MRtrix3 (Tournier et al., 2004, 2007, 2012). Briefly, the skull of the DWI dataset was removed and a brain mask was formed. A response function, representing the DW signal for a single fiber population, was estimated (using the "dwi2response" function in MRtrix3) and then incorporated into a non-negativity CSD analysis from which a FOD was computed for each WM voxel (using the "dwi2fod" function In MRtrix3). Finally, streamline tractography (using the "tckgen" function in MRtrix3) was performed with parameters initiated at stopping FA value $=0.15$, minimal length $=5 \mathrm{~mm}$, and step size $=$ $0.3 \mathrm{~mm}$. Graphical representations of this reconstruction method are detailed elsewhere (Tournier et al., 2012).

Figure 1 illustrates the differences between the three reconstruction methods under investigation in this manuscript. Table 2 details the tracking parameters used for each reconstruction method.

\section{Tractography Assessment Criteria}

The following criteria were used to assess each tractography method:

1) Model creation and tractography time: the duration of time for either tensor-based or FOD-based models to be constructed from preprocessed scans, and the duration of time for subsequent tractography analysis.

2) Anatomical accuracy of tractography output: whether generated WM tracts resembled known anatomical fiber organization.

3) Depiction of $\mathrm{CN}$ compression by tumor: whether it was possible to visualize where the tumor in the posterior cranial fossa was compressing the $\mathrm{CN}$ of interest.

Table 3 further specifies this assessment criteria. 


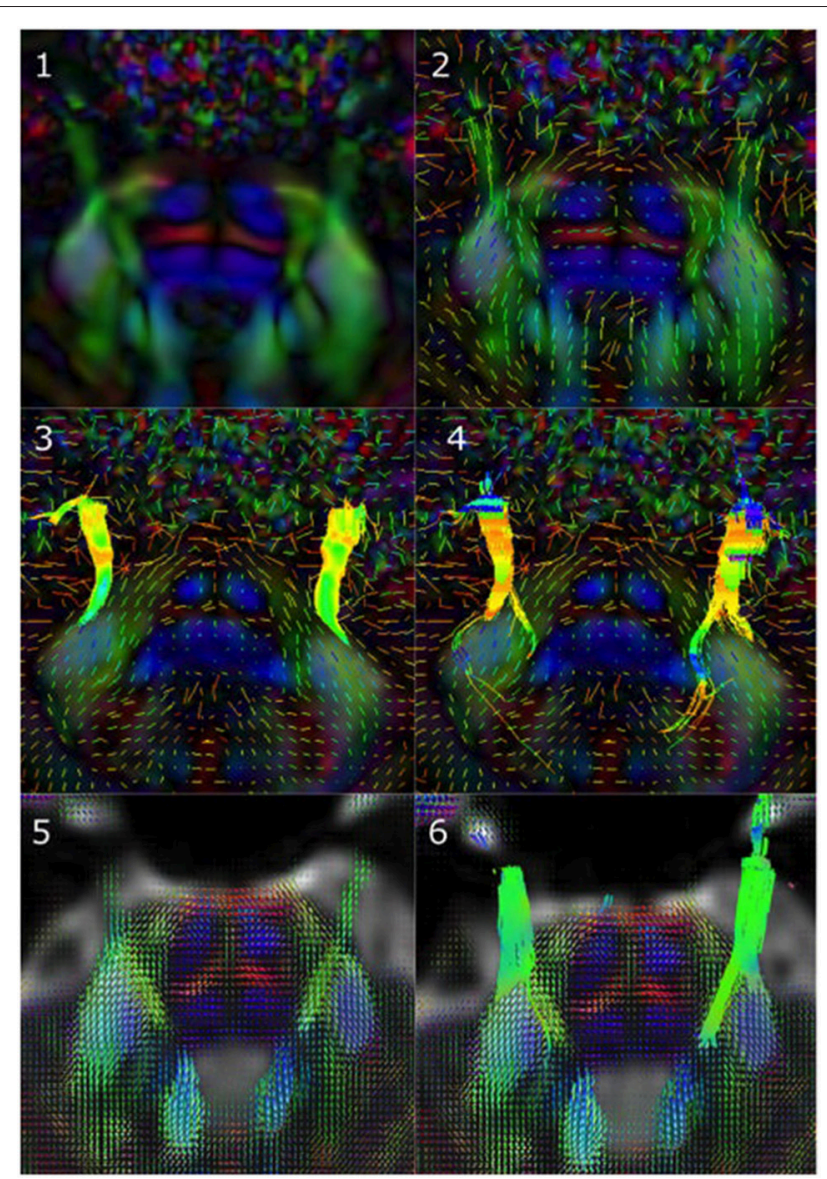

FIGURE 1 | Reconstruction methods differ in how they represent underlying diffusion processes. (1) Axial view of a sample tensor map displaying CN V entry into the brainstem, (2) Tensors visible as glyphs displaying CN V entry into the brainstem, (3) Results from single diffusion tensor tractography (SDT) after placing region-of-interest (ROI) seeds on anterior portions of $\mathrm{CN} \mathrm{V}$ bilaterally. Generated streamlines do not show their projections to brainstem nuclei, (4) Results from EXtended Streamline Tractography (XST) after placing $\mathrm{ROI}$ seeds on anterior portions of $\mathrm{CN} V$ bilaterally. Streamlines projecting to the area of the nuclei of the trigeminal nerves can be seen, (5) Axial view of sample fiber orientation distribution (FOD) map displaying CN V entry into the brainstem, (6) Results from streamline tractography on FODs derived from constrained spherical deconvolution (CSD) after placing $\mathrm{ROI}$ seeds on anterior portions of CN V bilaterally. Streamlines projecting to the area of the nuclei of the trigeminal nerves can be seen.

\section{Tumor Modeling}

T1 anatomical images were registered to the DWI using linear registration in $3 \mathrm{D}$ Slicer version 4.3 (NA-MIC $\left.^{(}\right)$. The registration procedure was assessed for accuracy in the brainstem area by ensuring that there was accurate alignment of 3 specific anatomical landmarks between DWI and T1 datasets: (a) basilar artery, (b) the ventral bulge of the basis pontis, and (c) the outline of the fourth ventricle.

With clear registration between $\mathrm{T} 1$ and DWI datasets achieved, a 3D tumor model was then created in 3D Slicer version 4.3. Briefly, the outline of the tumor was manually traced on each

TABLE 2 | Tracking parameters used for each reconstruction method.

\begin{tabular}{ll}
\hline $\begin{array}{l}\text { Reconstruction } \\
\text { method }\end{array}$ & Tracking parameters \\
\hline SDT & -stoppingvalue 0.15 -stopping curvature 0.8 -minimumlength \\
& 5 -clthreshold 0.15 -integrationsteplength 0.1 -seedspacing \\
& 0.3 \\
XST & -stop aniso:c1,0.1 -stop FA 0.15 -frac 0.1 -radius 0.8 -minlen \\
& 5 -step 0.3 \\
CSD & -algorithm SD_STREAM -step 0.3 -angle 45 -rk4 -minlength \\
& 5 -cutoff 0.15 -initcutoff 0.15 -force \\
\hline
\end{tabular}

TABLE 3 | Criteria for assessing the results obtained from the three discrete tractography methodologies.

\section{PROCESSING SPEED}

- Time required for generation of tensor or FOD model

- Time required for tractography once a suitable seed $\mathrm{ROI}$ is created

\section{ANATOMICAL ACCURACY}

\section{CN V}

- Adequate representation of cisternal segments of CN V

- Adequate representation of projections to brainstem CN V nuclei

- Overall accurate delineation of CN V

\section{CN VII/VIII}

- Adequate representation of cisternal segments of CN VII/VIII

- Overall accurate delineation of CN VIINIII

\section{RELATIONSHIP BETWEEN CN FIBER BUNDLES AND TUMORS IN}

\section{POSTERIOR CRANIAL FOSSA}

- Adequate visualization of fiber compression in all patients with tumors primarily compressing CN VII/NIII

- Adequate visualization of fiber compression in all patients with tumors primarily compressing CN V

axial slice. This volume was then converted to a $3 \mathrm{D}$ model using a Laplacian filter with 30 iterations.

All results from the three reconstruction methods were displayed on either T1 anatomical images or mixed anatomicaldiffusion tensor models, which included a $3 \mathrm{D}$ model of the posterior cranial fossa tumor. The mixed anatomical-diffusion tensor model represents the tensor map being overlaid on a $\mathrm{T} 1$ anatomical image. The results were imported into 3D Slicer for visualization. Importantly, no filtering was applied to the generated tracts. All computations were performed on a Dell Precision T3610 Desktop on a Ubuntu 12.04 LTS OS.

\section{RESULTS}

\section{Processing Time}

Imaging datasets including DWI scans from all 10 patients with cerebellopontine angle tumors were successfully corrected for motion-related artifacts. No image datasets were discarded for excessive head motion or associated distortions.

As processing time is a major concern for neurosurgeons hoping to incorporate DWI-based technology into their practice, we measured processing time for the following steps in this 


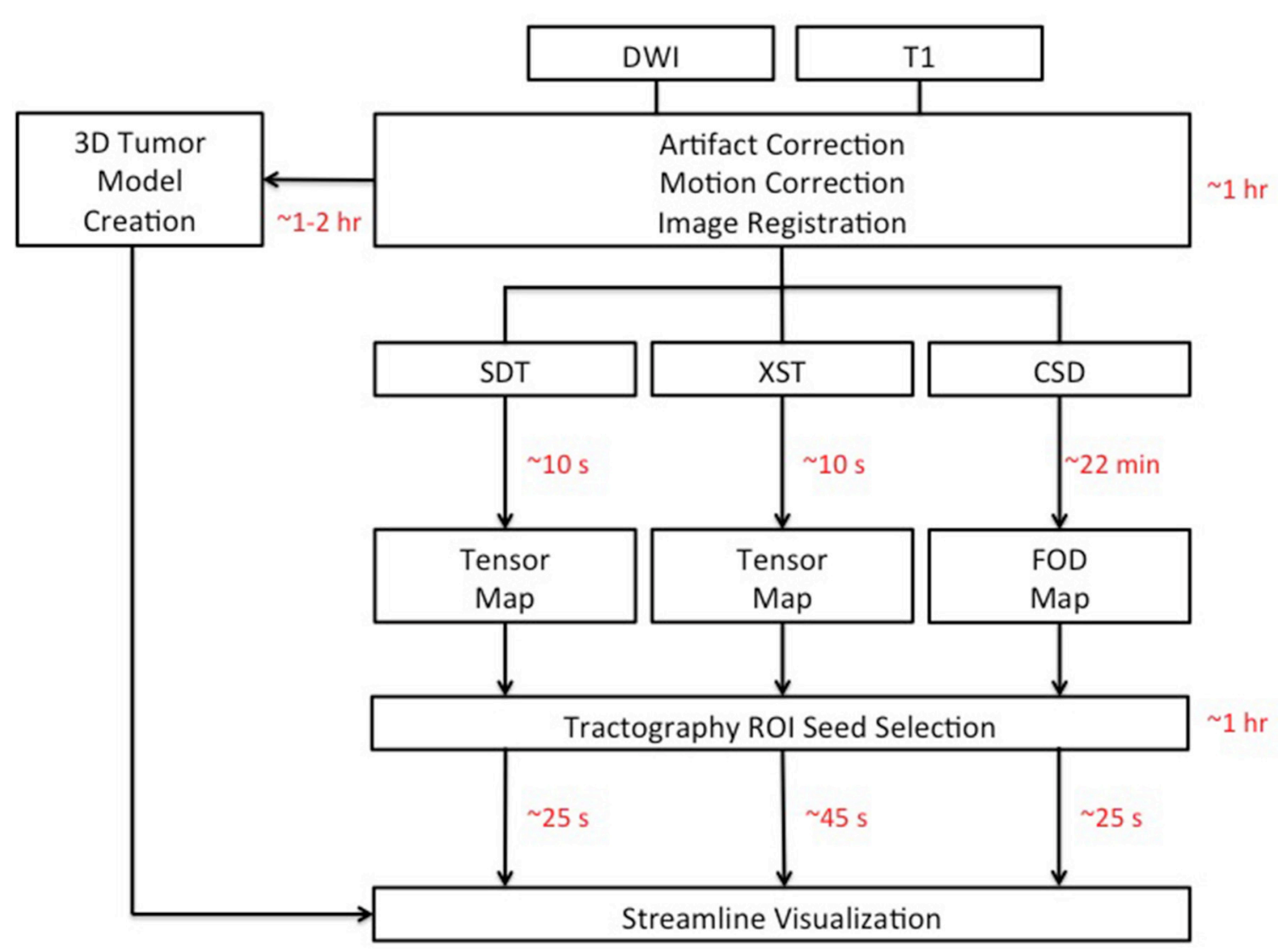

FIGURE 2 | Schematic detailing the analysis pipelines for all three tractography methods: Single Diffusion Tensor Tractography (SDT), EXtended Streamline tractography (XST), Streamline tractography on fiber orientation distributions (FODs) derived from constrained spherical deconvolution (CSD). Acquired diffusion-weighted imaging (DWI) scans were initially corrected for motion- and eddy current-related artifacts. This took $\sim 30$ minutes (min) to perform. Registration between T1 anatomical images and DWI images was complete within 1 hour (hr) per patient. 3D models of tumors of the posterior cranial fossa took 1-2 hr per patient to create. The creation of the tensor map was typically $\sim 10$ seconds (s) in duration, where the creation of the FOD map was typically $\sim 22$ min in duration. Tractography analysis for cranial nerves (CNs), not directly affected by the presence of a tumor, was complete within 10 min for CN V and 20 min for CN VII/ VIII, on average. Tractography analysis for CNs directly affected by the presence of a tumor took longer with 30 min on average for CN $\mathrm{V}$ generation in patients with tumors compressing CN V and 1-2 hr in patients with tumors compressing CN VII/VIII. Once suitable seed ROls had been created, SDT and CSD-based tractography took typically $\sim 25 \mathrm{~s}$ on average for CN generation with XST typically taking $\sim 45 \mathrm{~s}$ on average. DWI: Diffusion-Weighted Image.

procedure: (i) correcting for motion-related and eddy currentinduced artifacts, (ii) Registration between T1 anatomical and diffusion images for anatomical localization, (iii) 3D tumor modeling, (iv), diffusion model (either tensor or FOD) creation, (v) Appropriate seed selection, and (vi) the tracking algorithm itself. These steps, and their associated processing times, are depicted in Figure 2. Representative seeds for tractography are depicted in Figure 3.

Most processing time stemmed from ensuring accurate registration between diffusion and $\mathrm{T} 1$ images and accurate tumor model creation $[\sim 1$ and $1-2$ hours (hr) per patient, respectively]. Ensuring appropriate seed selection for $\mathrm{CN}$ VII/VIII in patients presenting with vestibular schwannomas (P05-P10) was also time-consuming (1-2 hr per patient for $\mathrm{P} 05-\mathrm{P} 10)$. In comparison, suitable seed selection for $\mathrm{CN} \mathrm{V}$ in patients with tumors primarily compressing this nerve (P01P04) took $\sim 30$ min per patient, indicating that the type of cerebellopontine angle tumor can have a sizeable impact on the time taken for appropriate seed selection. Seed selection for $\mathrm{CN} \mathrm{V}$ and $\mathrm{CN}$ VII/VIII on sides that were not directly affected by the presence of a tumor took $\sim 10$ and $20 \mathrm{~min}$, respectively. Correction of motion-related and other image artifacts had an approximate duration of $30 \mathrm{~min}$ in total, per patient.

There were also notable processing time differences between the three reconstruction methods. The creation of the tensor map was rapid [typically 10 seconds (s)], compared to the running of the constrained spherical deconvolution resulting in fiber orientation distribution estimates (typically $22 \mathrm{~min}$ ). For tractography itself, once a suitable seed ROI had been selected, XST took slightly longer for streamline generation (typically $\sim 45 \mathrm{~s}$ for $\mathrm{CN}$ generation), when compared to both SDT and CSD-based reconstruction methods (typically $\sim 25 \mathrm{~s}$ for $\mathrm{CN}$ generation from either method). 


\section{Anatomical Accuracy CN V}

Figure 4 depicts $\mathrm{CN} \mathrm{V}$ generated from each of the three reconstruction methods for all 10 patients with tumors of the cerebellopontine angle. All three reconstruction methods accurately delineated portions of $\mathrm{CN} \mathrm{V}$, particularly the cisternal and retrogasserian segments. However, there were notable differences between streamlines generated from each method, in terms of accuracy and aberrant streamline generation. While SDT accurately portrayed cisternal portions of $\mathrm{CN} \mathrm{V}$, its accuracy diminished closer to the brainstem with its inability to show projections to trigeminal brainstem nuclei and instead solely generated superior cerebellar peduncle streamlines (e.g., Figure 4, P03-SDT). Both XST and CSD-based tractography more accurately demonstrated the distinction between superior cerebellar streamlines and projections to trigeminal brainstem nuclei (Figure 4, P03-XST and CSD). Similarly, SDT sometimes produced inaccurate projections into the cerebellum when tracking superior cerebellar streamlines (Figure 4, P09-SDT). CSD-based reconstruction methods also sometimes produced similar erroneous cerebellar projections (Figure 4, P09 CSDx).

\section{CN VII/VIII}

Figure 5 depicts CN VII/VIII generated from each of the three reconstruction methods. Similarly to $\mathrm{CN} \mathrm{V}$, all three methods successfully portions of CN VII/VIII, particularly the cisternal segment, with XST and CSD-based reconstruction methods tending to generate longer portions of the CNs, particularly CNs VII/VIII that were not in the proximity of a tumor, when compared to SDT (Figure 5, P02, P03, P04, P06, P10).

On the other hand, while CSD-based reconstruction methods were able to provide detailed reconstructions of the CNs, it sometimes generated a notable amount of spurious streamlines, when compared to the output from either other method. The depictions of CN VII/VIII in P08 illustrate this point (Figure 6). CSD-based reconstruction methods produced several aberrant streamlines that emerged superiorly, anteriorly and posteriorly from the generated cisternal segment of CN VII/VIII, between its exit from the brainstem and where it came into contact with tumor, compared to the two other methods.

\section{Depiction of Tumor/CN Relationship CN V}

All three methods satisfactorily illustrated this relationship in the four patients presenting with tumors primarily affecting $\mathrm{CN} \mathrm{V}$ (Figure 4, P01, P02, P03, P04). It was possible to clearly see where the generated $\mathrm{CN} \mathrm{V}$ came into contact with the generated $3 \mathrm{D}$ tumor model and would have allowed for clear tumor resection borders to be defined.

\section{CN VII/VIII}

Similarly, it was possible to visualize where the lesion was compressing CN VII/VIII, using all three methods under consideration, in most of the patients presenting with vestibular schwannomas (Figure 5, P05, P07, P08, P09, P10). Notably, in one patient (Figure 7, P06), only XST and CSD-based reconstruction methods could depict CN VII/VIII curving under
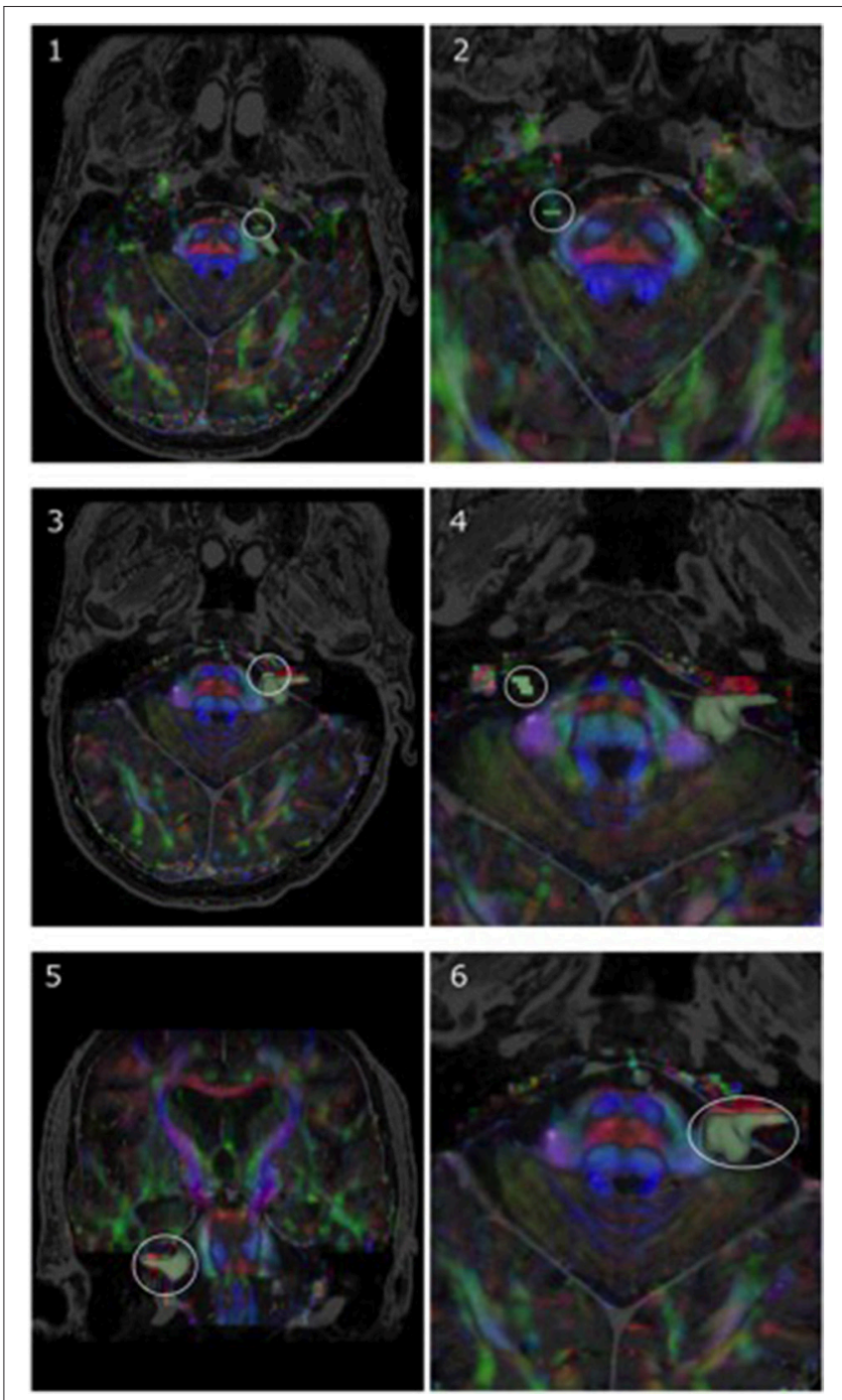

FIGURE 3 | Representative region-of-interest (ROI) seed placement, in green, in one patient with a vestibular schwannoma (P05). (1, 2) Highlight ROls placed on anterior portions of $\mathrm{CN} \mathrm{V}$, whereas $(3,4)$ specify the ROI location for $\mathrm{CN}$ VII/NIII on both the affected side and side contralateral to the lesion, respectively. Images are displayed on a mixed anatomical-tensor model from a superior view. The mixed anatomical-diffusion tensor model represents the tensor map being overlaid on a T1 anatomical image. Pictures of the vestibular schwannoma from both a coronal perspective (5) and zoomed-in axial perspective (6) are also displayed.

the posterior cranial fossa tumor. SDT was not able to do so and simply displayed a portion of CN VII/VIII anterior to the tumor and not curving underneath it.

Table 4 illustrates results, for each tractography method, in relation to the tractography assessment criteria.

\section{DISCUSSION}

XST appears to be the optimal reconstruction method for $\mathrm{CN}$ generation in a neurosurgical context, due to its speed 


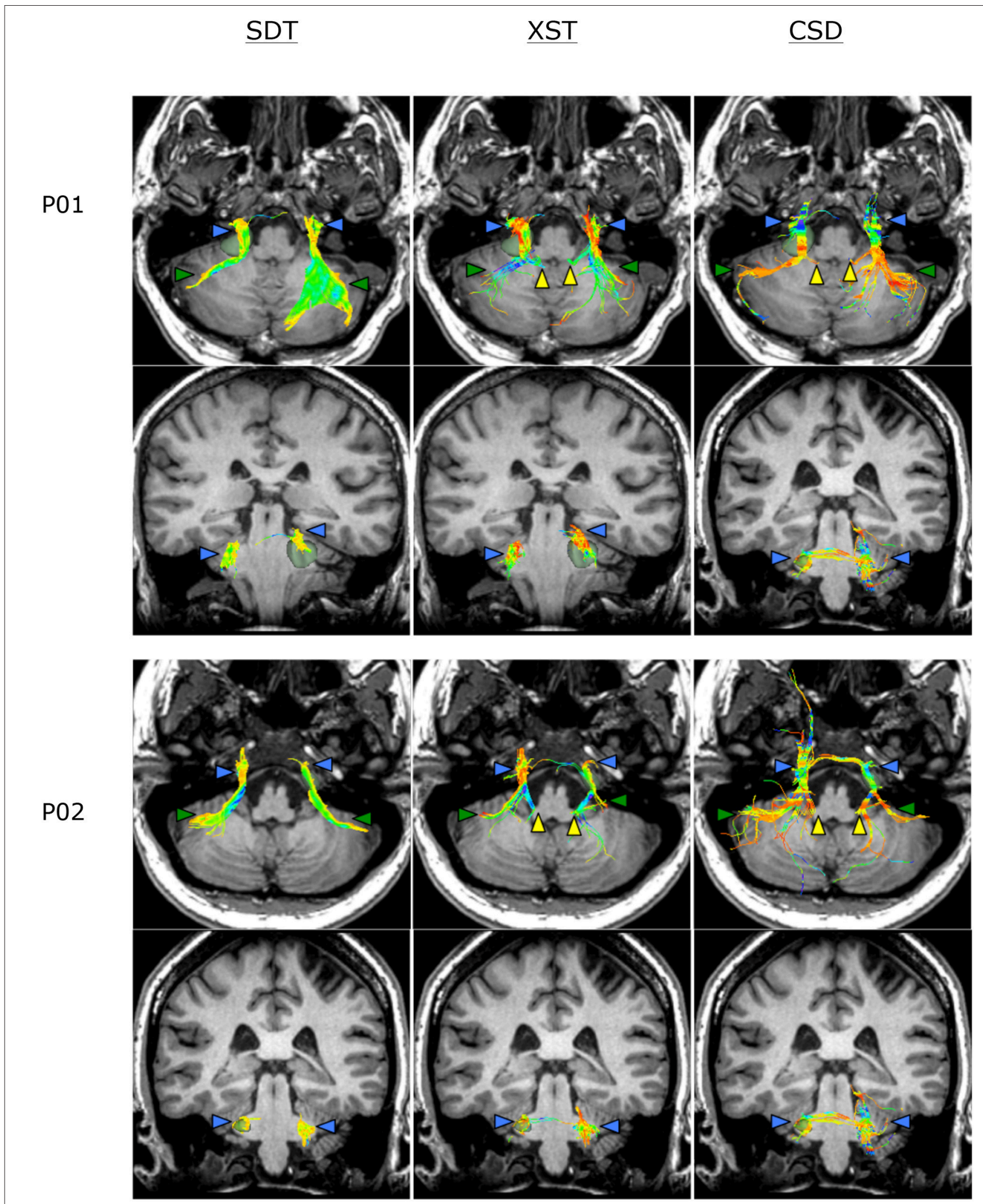

FIGURE 4 | Continued 


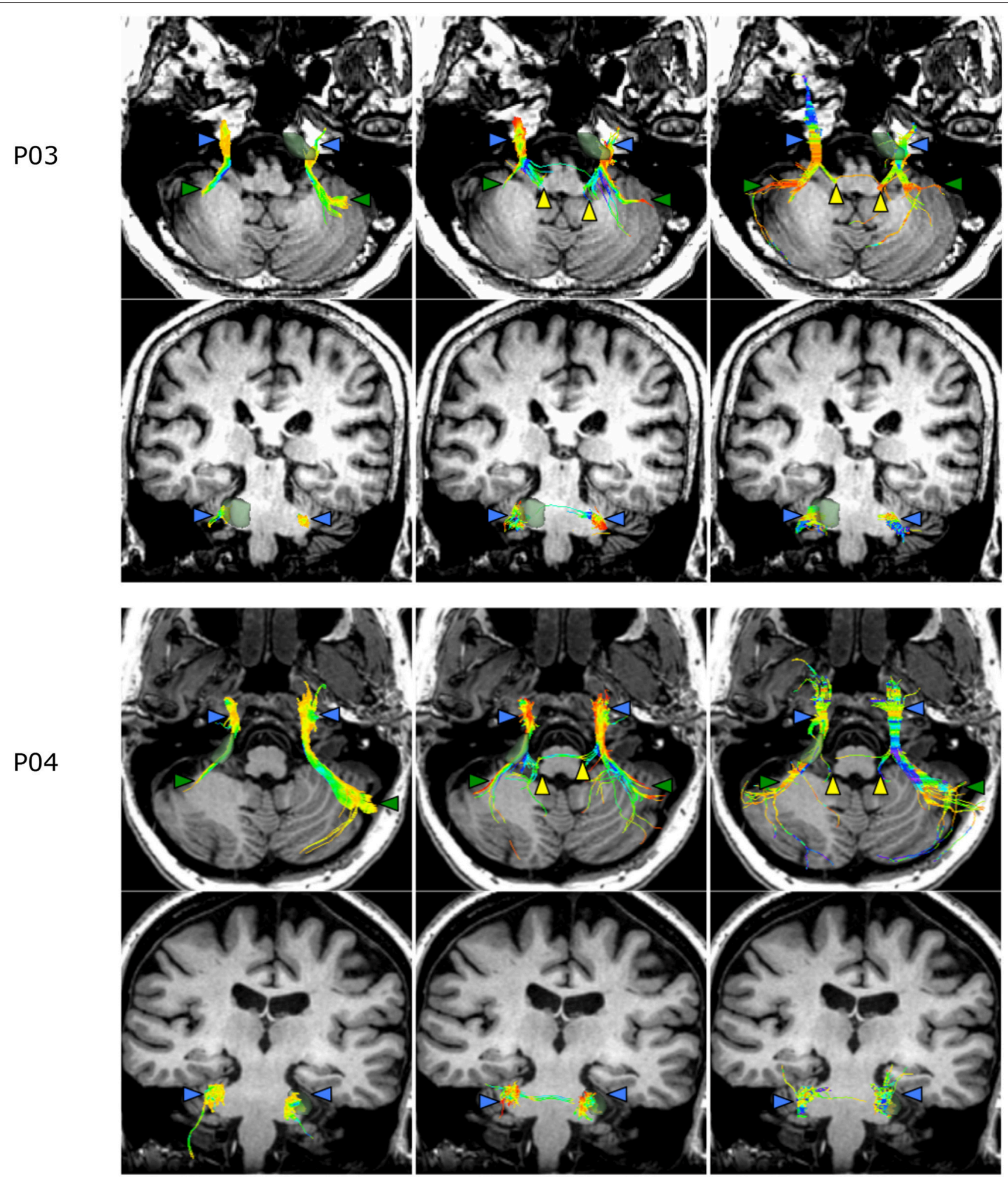

FIGURE 4 | Continued 


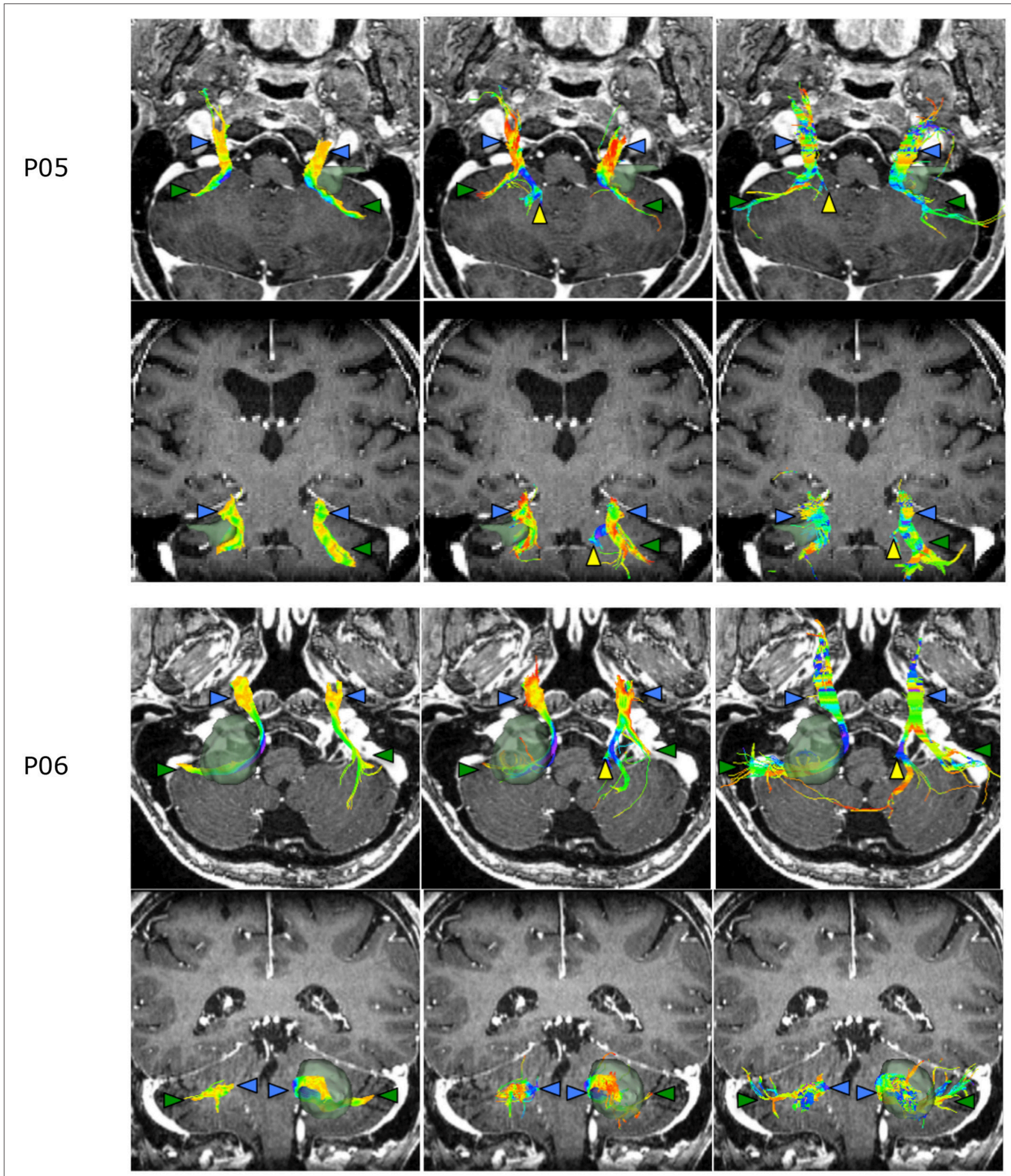

FIGURE 4 | Continued 


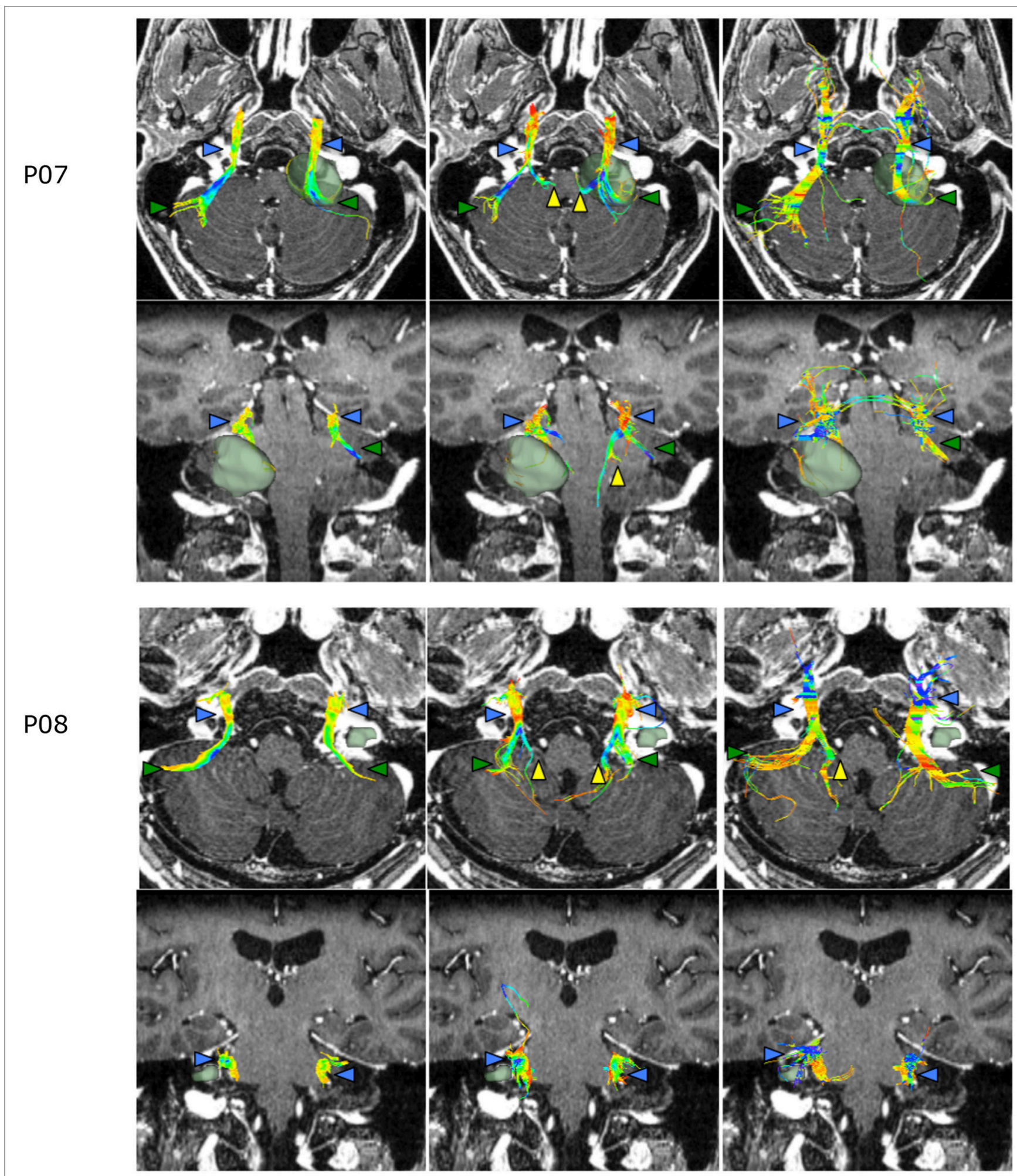

FIGURE 4 | Continued 

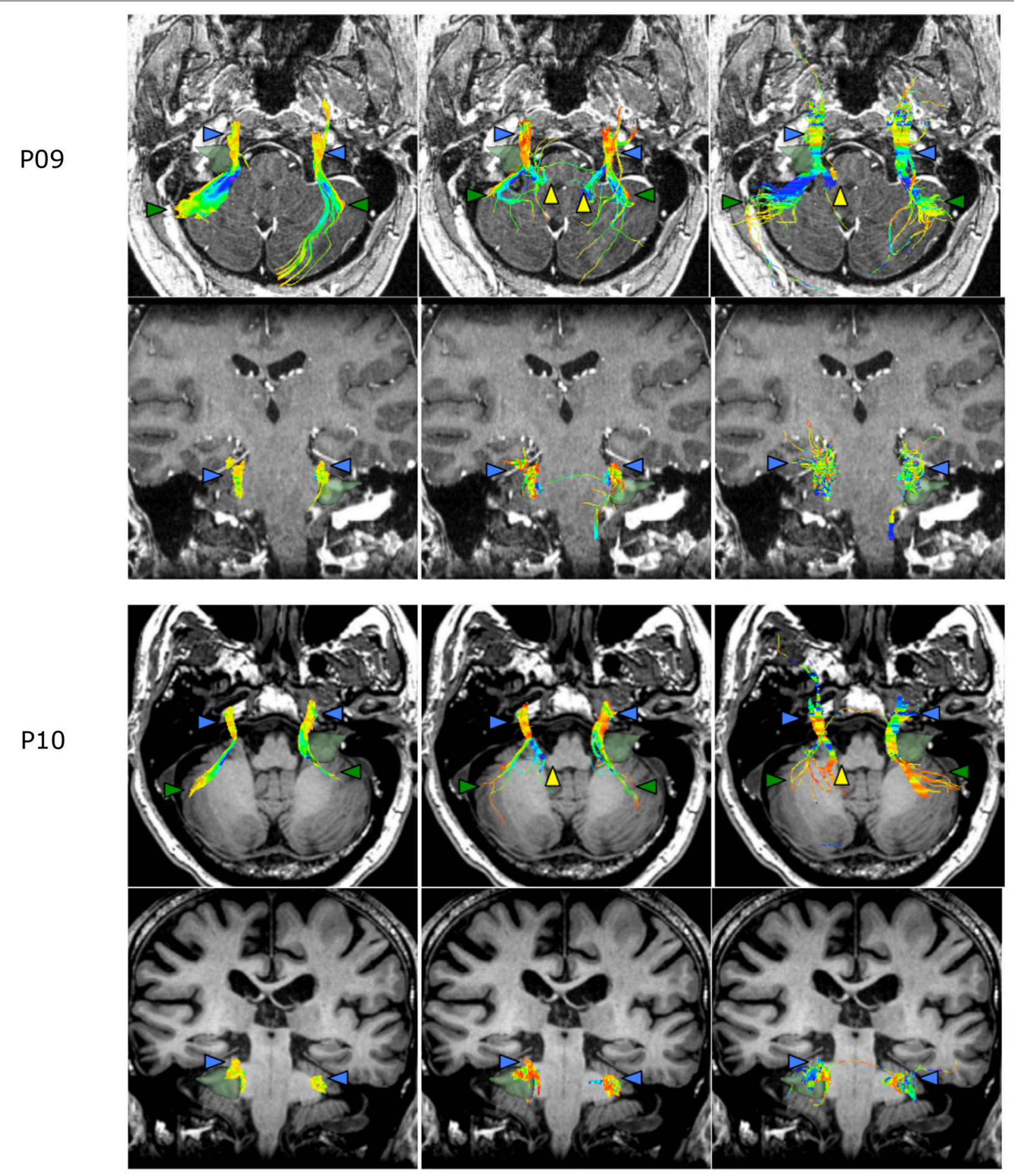

FIGURE 4 | Bilateral CN V generated from each of the three reconstruction methods: Single Diffusion Tensor Tractography (SDT), EXtended Streamline tractography (XST), and streamline tractography on fiber orientation distributions (FODs) derived from constrained spherical deconvolution (CSD) is displayed. Streamlines are displayed overlaid on a T1 anatomical image from a superior and anterior view. Colored triangles indicate particular anatomical landmarks: blue indicates cranial nerves, green indicates superior cerebellar fibers, yellow indicates trigeminal brainstem nuclei. While SDT produced good representations of cisternal segements of CN V (P05-SDT), it was unable to display projections to trigeminal brainstem nuclei (P03-SDT). Both XST and CSD-based tractography could differentiate between superior cerebellar peduncle fibers and trigeminal brainstem nuclei (P03-XST, CSD). SDT also generated aberrant broad projections into the cerebellum when imaging superior cerebellar peduncle fibers (P09-SDT). CSD-based tractography also produced several aberrant fibers when generating superior cerebellar peduncle fibers that encounter trigeminal fibers (P09-CSD). 


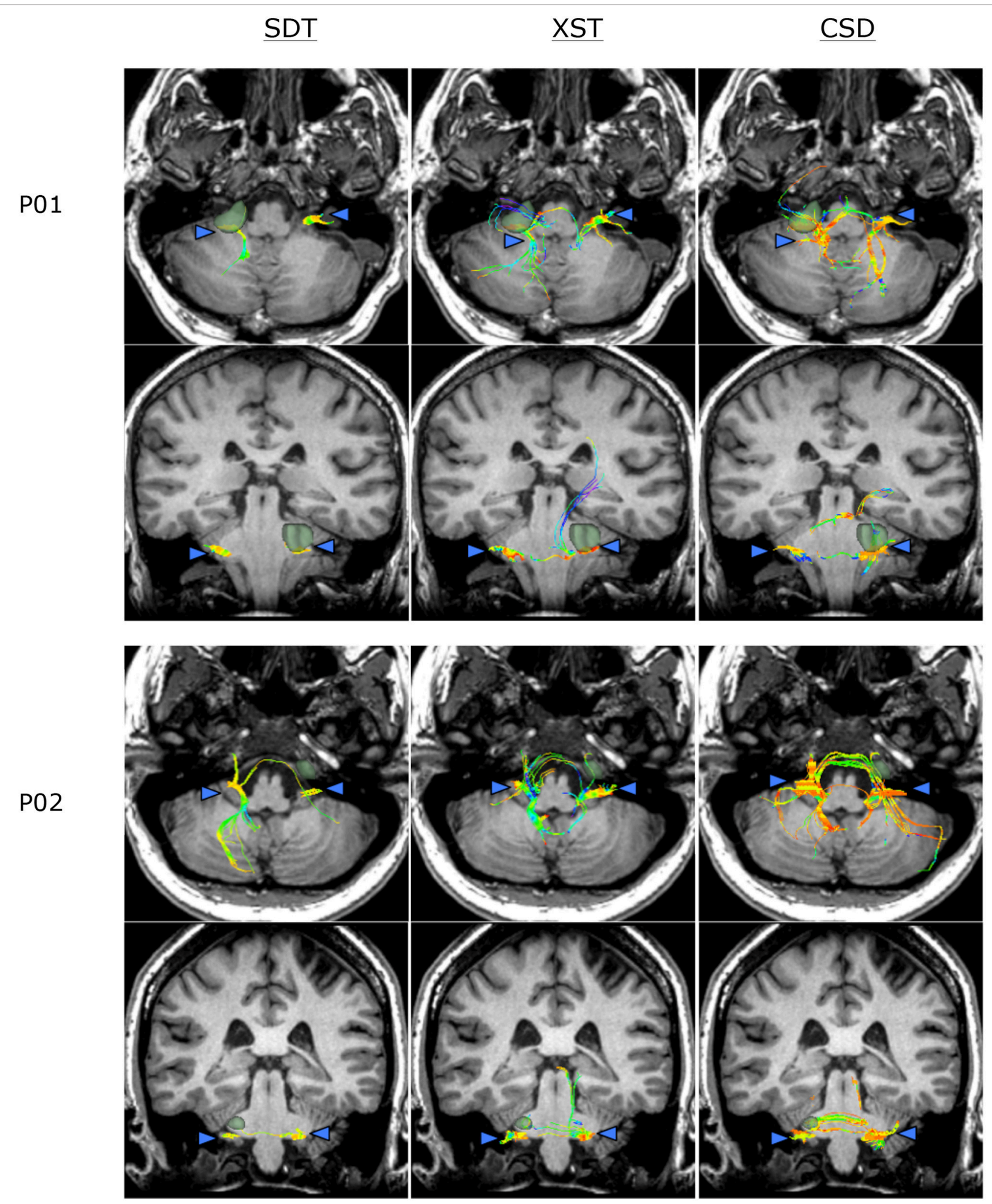

FIGURE 5 | Continued 


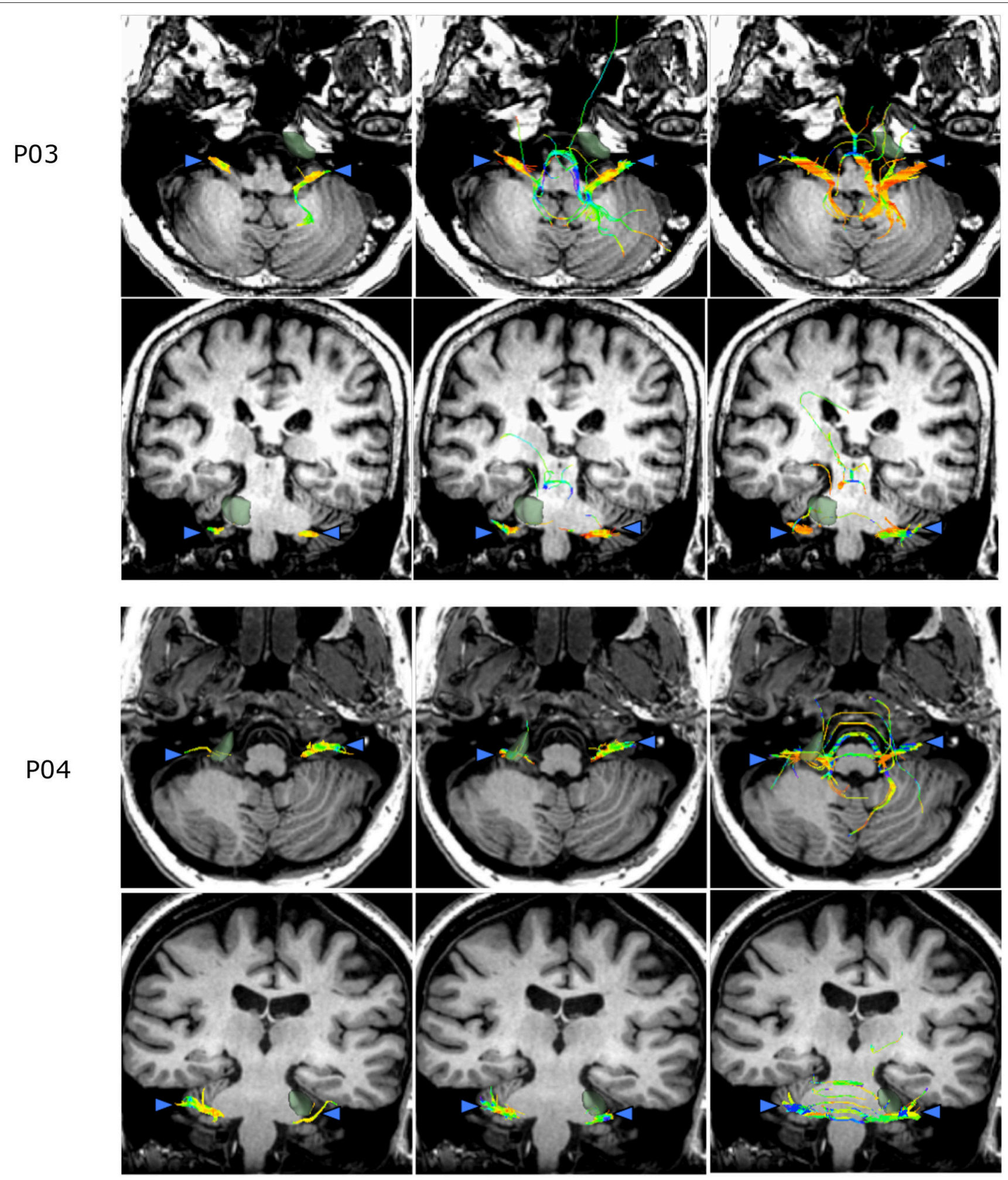

FIGURE 5 | Continued 

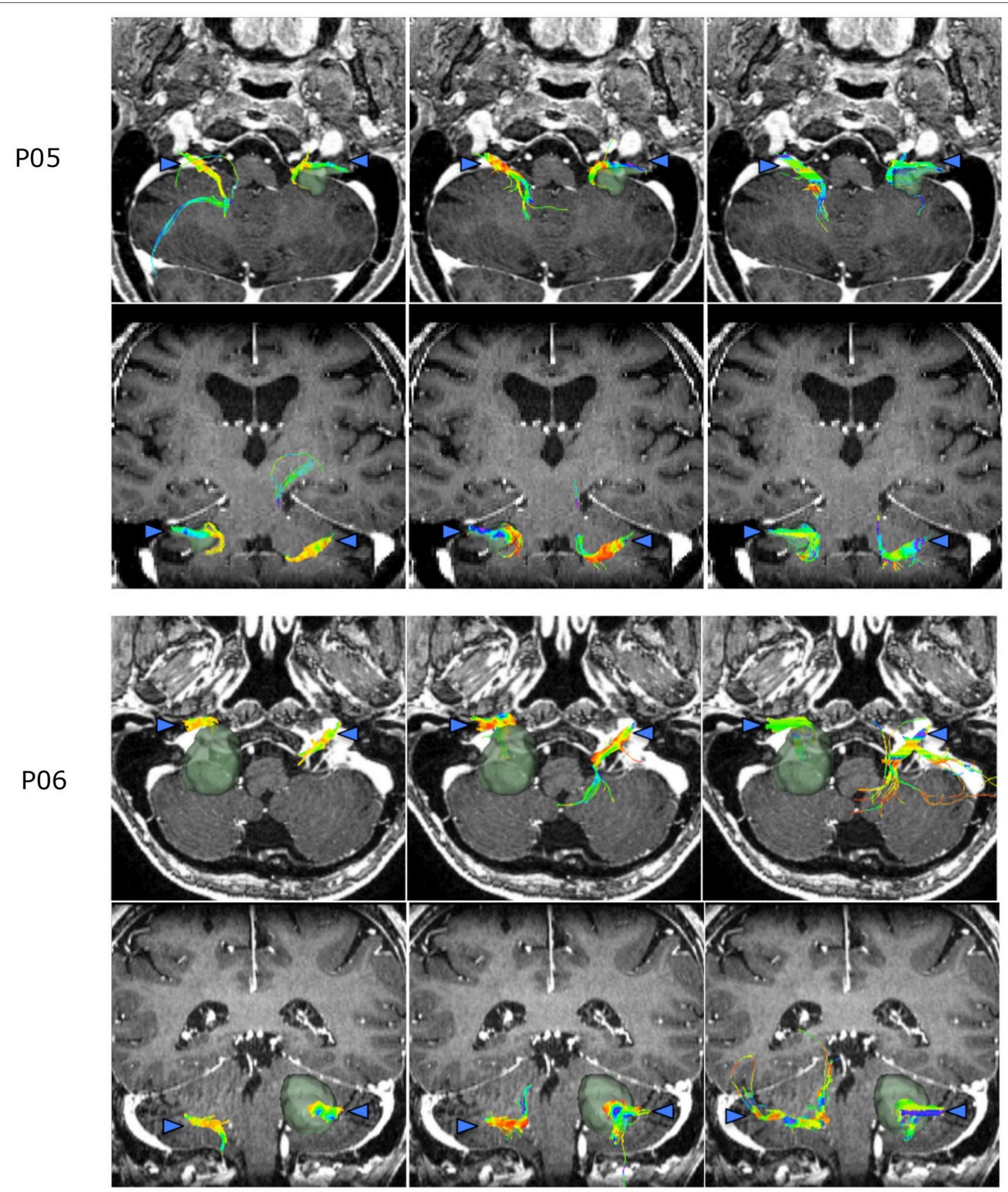

FIGURE 5 | Continued 


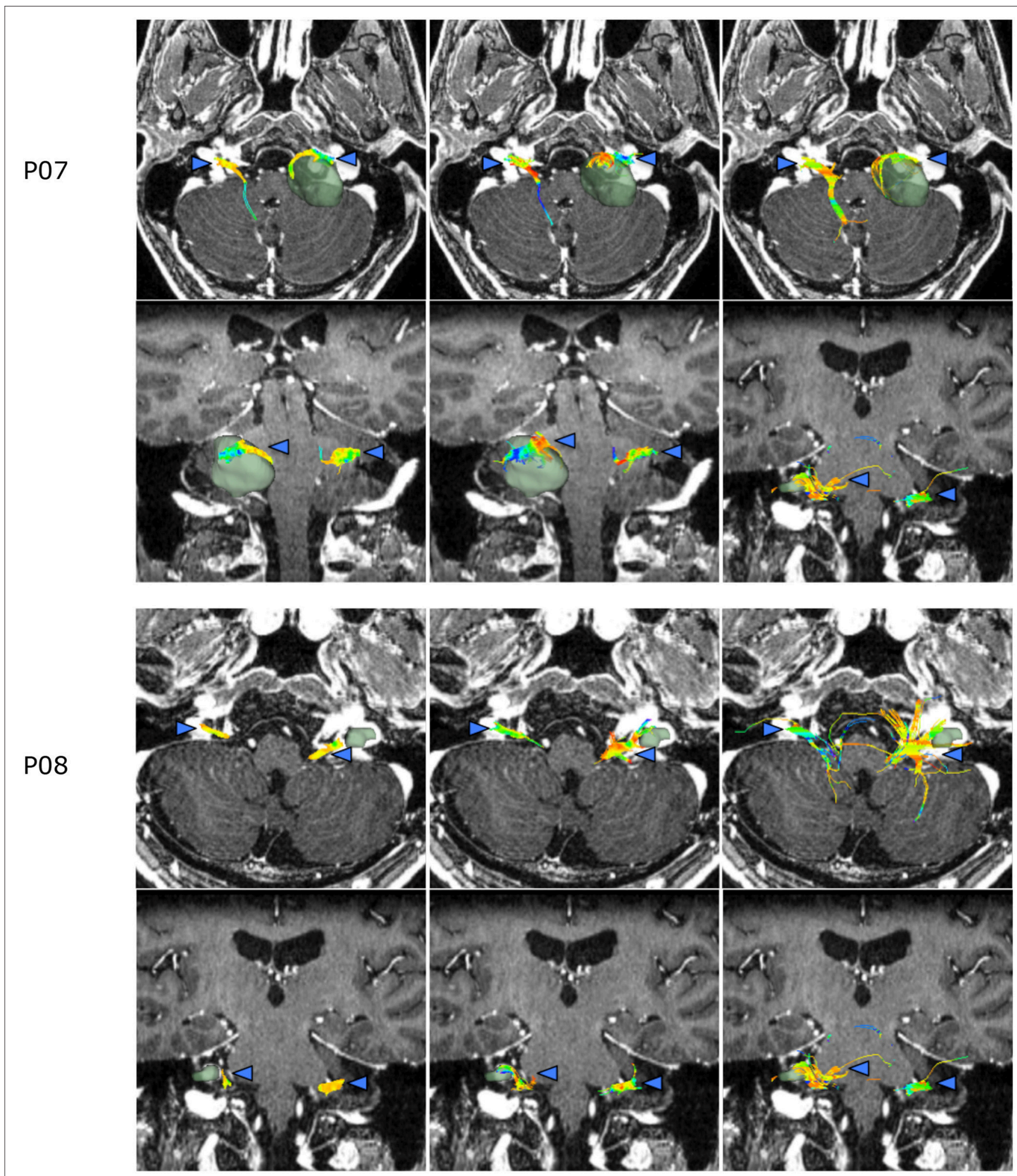

FIGURE 5 | Continued 

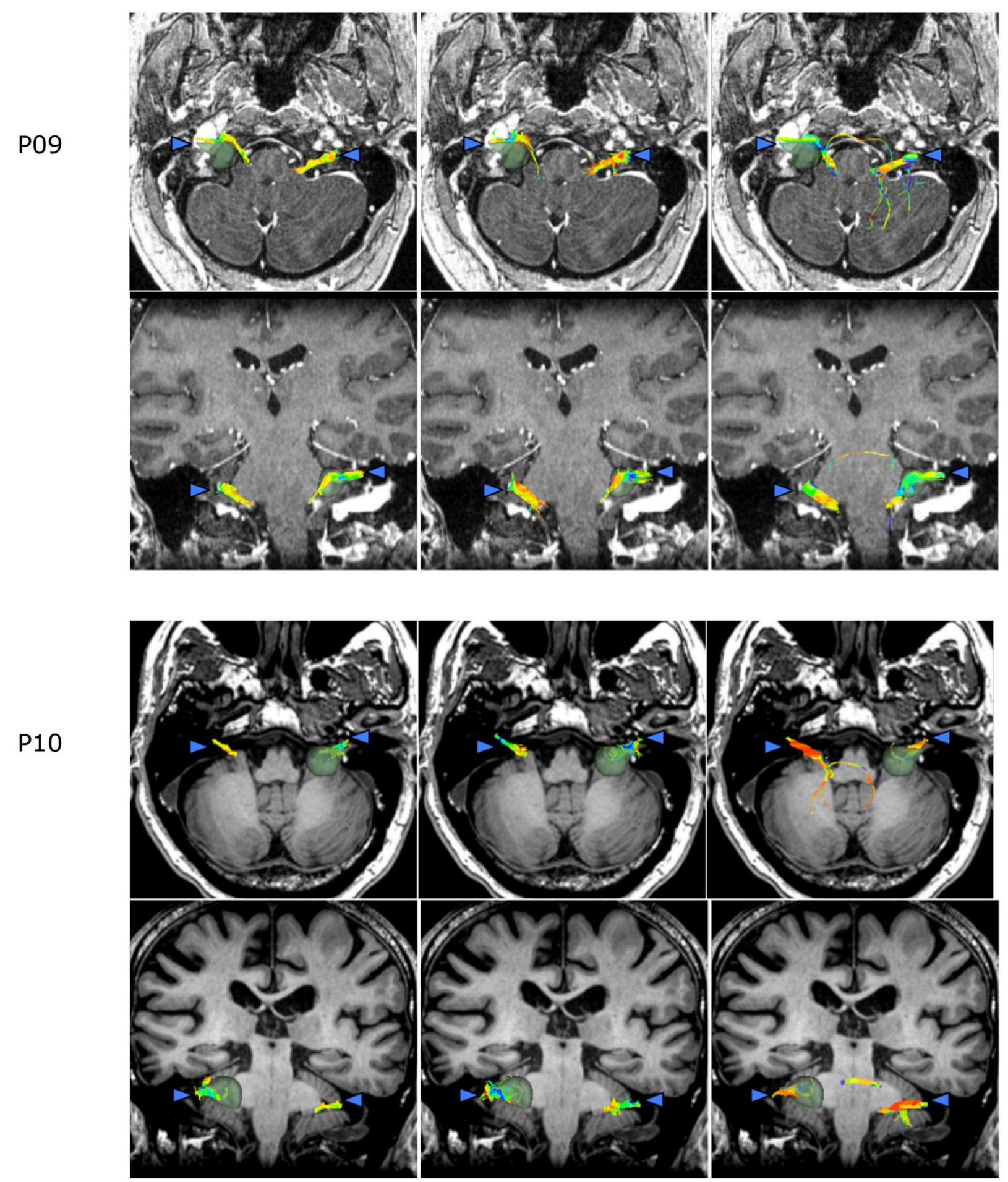

FIGURE 5 | Bilateral CN VIINIII generated from each of the three reconstruction methods: Single Diffusion Tensor Tractography (SDT), EXtended Streamline tractography (XST), Streamline tractography on fiber orientation distributions (FODs) derived from constrained spherical deconvolution (CSD) is displayed. Streamlines are displayed overlaid on a T1 anatomical image from a superior and anterior view. Colored triangles indicate particular anatomical landmarks: blue indicates cranial nerves, green indicates superior cerebellar fibers, yellow indicates trigeminal brainstem nuclei. SDT was able to display anatomically accurate cisternal portions of CN VII/VIII on sides contralateral to lesions (P06-SDT). However, XST and CSD-based tractography produced longer sections of CN VII/VIII (P10-SDT, XST, CSD). On affected sides, SDT could only generate small cisternal segments (P08-SDT). XST and CSD-based tractography depicted longer cisternal segments (P08-XST, CSD). However, CSD-based tractography also generated several anatomically inaccurate fibers when generating CN VII/VIII in this patient (P08-CSD) 


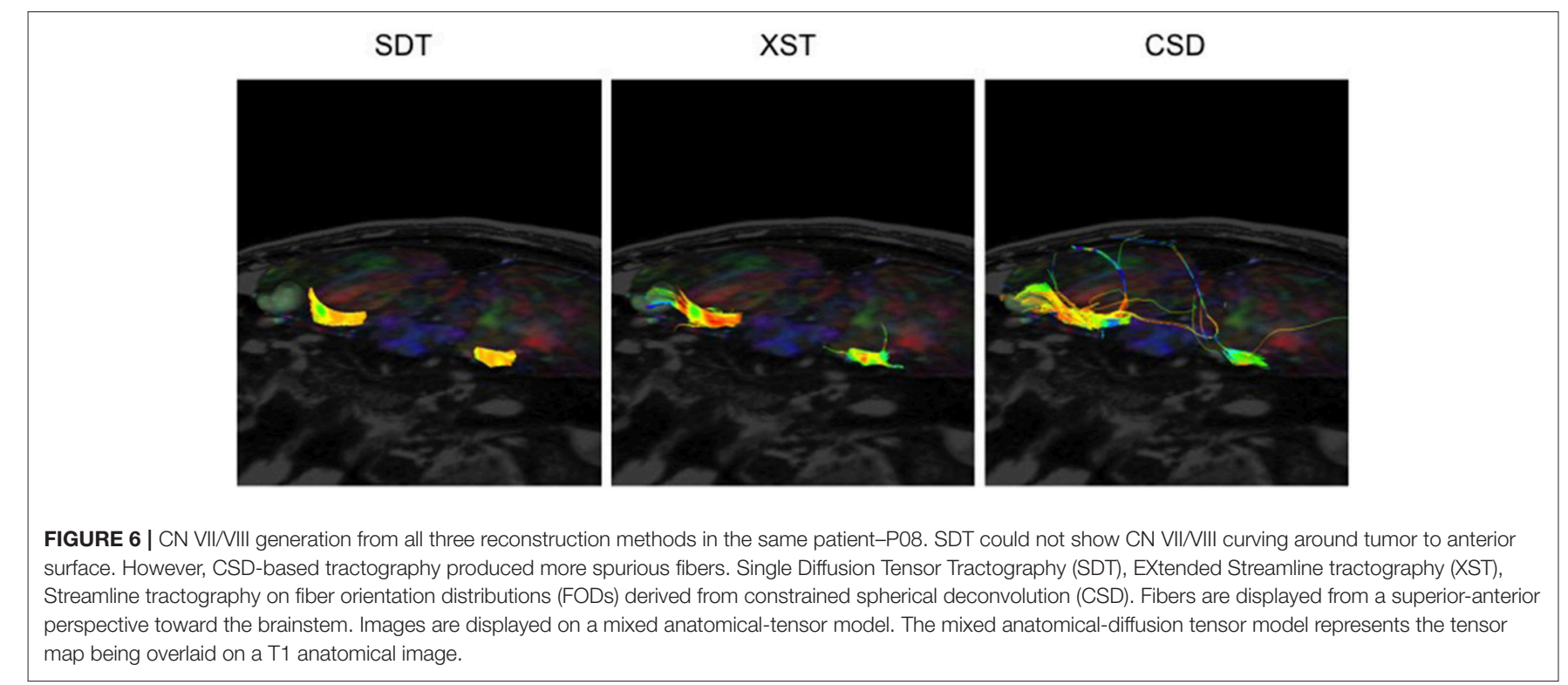

SDT
FIGURE 7 I CN VIIVIII generation from all three reconstruction methods in a patient with a vestibular schwannoma compressing CN VII/VIII-P06. SDT was only able
to show portions of CN VII/VIII anterior to the tumor, where both XST and CSD-based tractography could show CN VII/VIII curving under the vestibular schwannoma.
Single Diffusion Tensor Tractography (SDT), EXtended Streamline tractography (XST), Streamline tractography on fiber orientation distributions (FODs) derived from
constrained spherical deconvolution (CSD). Fibers are displayed from a posterior-lateral perspective toward the brainstem. Images are displayed on a mixed
anatomical-tensor model. The mixed anatomical-diffusion tensor model represents the tensor map being overlaid on a T1 anatomical image.

and accuracy, when compared to the other methods under consideration here. XST uses the conventional tensor map and is nearly as rapid as the other techniques under investigation here in terms of tracking time. XST was able to generate larger anatomically accurate portions of the $\mathrm{CN}$ of interest, compared to SDT-the most commonly used reconstruction method for neurosurgical purposes. Further to this, it also could differentiate representations of fiber tracts, while not generating as many aberrant streamlines, as CSD-based streamline tractography did.

Here, we found that while SDT was the most accessible and easy-to-use program (i.e., tracking can be performed through a Graphical User interface) of all three reconstruction methods under review, its results oftentimes only displayed smaller portions of the $\mathrm{CNs}$ of interest, compared to the two other methods under consideration and could not distinguish between representations of $\mathrm{CN}$ and cerebellar peduncle fibers. Such inaccurate results from SDT reconstruction methods may pose a major problem for neurosurgeons who would need to know exactly where $\mathrm{CN} \mathrm{V}$ exits the brainstem and the relationship between the $\mathrm{CN}$ itself and nearby cerebellar peduncular fibers. Also, in one patient with a vestibular schwannoma, SDT reconstruction methods could not adequately depict the relationship between CN VII/VIII and tumor, where the other methods were able to do so. This ability to depict CN VII/VIII compression may be related to how both XST and CSD-based methods can adequately account for crossing fiber populations, where SDT cannot. Thus, this complex fiber arrangement of $\mathrm{CN}$ VII/VIII emerging from under the posterior cranial fossa tumor and projecting laterally to the cochlear region is only visualized from employing more advanced reconstruction methods. SDT, 
TABLE 4 | Summary of the results from the three tractography methodologies under consideration.

\begin{tabular}{llc}
\hline Methods & SDT & XST \\
\hline PROCESSING SPEED & $10 \mathrm{sec}$ & \multicolumn{1}{c}{ CSD } \\
\hline Time required for generation of tensor or FOD model & $25 \mathrm{sec}$ & $22 \mathrm{~min}$ \\
Time required for tractography once a suitable seed ROI is created & Yes \\
ANATOMICAL ACCURACY & No & No \\
CN V & No & Yes \\
Adequate representation of cisternal segments of CN V & Yes \\
Adequate representation of projections to brainstem CN V nuclei & Yes & Yes \\
Overall accurate delineation of CN V & No \\
CN VII/VIII & Yes \\
Adequate representation of cisternal segments of CN VII/VIII & Yes \\
Overall accurate delineation of CN VII/VIII & Yes \\
RELATIONSHIP BETWEEN CN FIBER BUNDLES AND TUMORS IN POSTERIOR CRANIAL FOSSA & Yes \\
Adequate visualization of fiber compression in all patients with tumors primarily compressing CN VII/VIII & Yes \\
Adequate visualization of fiber compression in all patients with tumors primarily compressing CN V & Yes \\
\hline
\end{tabular}

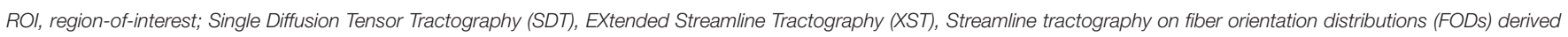
from constrained spherical deconvolution (CSD).

with its inherent assumption of one fiber bundle per voxel, could not adequately image the course of this fiber bundle as it curved under the tumor and should be a cautionary note for neurosurgeons implementing SDT through available software programs in their clinical practice.

Such complex fiber arrangements appear to be problematic for SDT to resolve, with its assumption of one fiber orientation per voxel. Previous work has illustrated such difficulties in patients with tumors in other parts of the brain. Kuhnt et al. (2013a) reported that SDT could not adequately resolve fibers in the vicinity of gliomas occurring in language-associated cortical areas. Similarly, Kuhnt et al. (2013b) observed that optic radiation fibers were not accurately depicted when SDT was applied to diffusion MRI data derived from patients with gliomas in the temporal lobe. Chen et al. (2015) demonstrated that disruptions to the arcuate fasciculus were not adequately illustrated using SDT in patients with tumors with peritumoral edema in language-related areas. Anatomically inaccurate depictions of corticospinal tracts derived from SDT in patients with tumors in the vicinity of the motor cortex have also been well described (Qazi et al., 2009; Farquharson et al., 2013; Chen et al., 2016).

Interestingly, while these limitations of the tensor model have been acknowledged for some time and how they may result in false tracking results, most software programs offering tractography tend to still mostly depend solely on it (Soares et al., 2013). This is since alternative HARDI algorithms, which can accommodate crossing fiber populations, require longer DWI scans with a large number of directions, which may be difficult to routinely implement in a clinical environment. Further to this, the application of advanced methods comes with its own set of challenges including the correction of distortion effects (Nimsky, 2014; Nimsky et al., 2016). The DWI scans used in this study, which allowed for implementation of HARDI algorithms, did not require a relatively large amount of time for acquisition
( $\sim 17.5 \mathrm{~min}$ in duration) and were collected as part of standard clinical protocol. This may be attractive to other neurosurgical groups interested in implementing HARDI-based tractography approaches.

Nonetheless a common consensus is still lacking as to which HARDI algorithm offers the best trade-off between scan acquisition time and tractography output for a clinical population. It appears that streamline tractography involving FODs derived from a CSD approach are demonstrating superior results compared to other HARDI-based models, such as qball imaging and "ball and stick" models, for larger WM pathways at least (Wilkins et al., 2015). However, we found that when it comes to smaller WM tracts, a multi-tensor reconstruction method, XST, appears to produce more reliable tracts from a conventional tensor approach-an approach that is widely available to neurosurgeons through currently accessible DWI software programs and takes a small amount of time (typically $10 \mathrm{~s}$ in this study) to compute. This reliability may stem how its propagation phase operates-the tensor, whose principal eigenvector has the least deviation from the incoming trajectory, is chosen for the next step during reconstruction. This approach helps ensure there is a consistent streamline trajectory, particularly when intravoxel multiple fibers are encountered.

It was interesting to note that while both XST and CSDbased reconstruction methods used different approaches, tensorand FOD-based ones, respectively, they shared similar outputs. Both were able to differentiate between $\mathrm{CN} \mathrm{V}$ and cerebellar tracts in a number of patients and clearly define where $\mathrm{CN}$ $\mathrm{V}$ exited the brainstem. In all cases, both methods were able to demonstrate the relationship between the posterior cranial fossa and affected CN. However, XST produced far fewer spurious streamlines than CSD-based streamline tractography leading to our recommendation that it should be the HARDI algorithm of choice for neurosurgical groups aiming to generate $\mathrm{CNs}$ in patients with posterior cranial fossa tumors. Previous 
demonstrations of CSD-based tractography have tended to involve probabilistic-based algorithms (Farquharson et al., 2013; Palesi et al., 2015). Here, we opted to use the CSD-based deterministic tractography, due to its superior processing speed for neurosurgical purposes (Qazi et al., 2009), which may explain the occurrence of these spurious fibers. While we acknowledge that it is possible to limit the number of tracks generated from CSD-based streamline tractography, as well as creating inclusion/exclusion ROIs, this raises other issues about choosing appropriate thresholds and criteria, all of which can take away from optimal tracking in a clinical setting, where rapid processing and interpretation are essential. Here, XST provided clear depictions of CNs without a reliance on the inclusion of extra criteria, in addition to those specified in the initial tractography command, making it an attractive option for neurosurgeons hoping to implement HARDI-based approaches in a time-dependent manner. As such reconstruction methods continue to be integrated in neurosurgical settings (Sammartino et al., 2016; Essayed et al., 2017), the selection of appropriate methods for accurate visualization of nerve fibers will continue to be of paramount importance.

\section{CONCLUSIONS}

These results suggest that a HARDI-based reconstruction method, XST, is currently the optimal option for visualizing how CNs $\mathrm{V}$ and VII/VIII are affected by the development of a tumor in the posterior cranial fossa. While the other reconstruction methods provide reasonable results, XST allows for a rapid tracking procedure where crossing fiber populations can be adequately represented while minimizing the number of anatomically inaccurate fiber representations

\section{REFERENCES}

Abdullah, K. G., Lubelski, D., Nucifora, P. G., and Brem, S. (2013). Use of diffusion tensor imaging in glioma resection. Neurosurg. Focus 34:E1. doi: 10.3171/2013.1.FOCUS12412

Basser, P. J., Mattiello, J., and Lebihan, D. (1994). MR diffusion tensor spectroscopy and imaging. Biophys. J. 66, 259-267.

Basser, P. J., Pajevic, S., Pierpaoli, C., Duda, J., and Aldroubi, A. (2000). In vivo fiber tractography using DT-MRI data. Magn. Reson. Med. 44, 625-632. doi: 10. 1002/1522-2594(200010)44:4<625::AID-MRM17>3.0.CO;2-O

Behrens, T., and Johansen-Berg, H. (2009). Diffusion MRI: from Quantitative Measurement to in-vivo Neuroanatomy. London: Elsevier.

Chen, D. Q., Quan, J., Guha, A., Tymianski, M., Mikulis, D., and Hodaie, M. (2011). Three-dimensional in vivo modeling of vestibular schwannomas and surrounding cranial nerves with diffusion. Neurosurgery 66, 788-795. doi: 10.1227/NEU.0b013e31820c6cbe

Chen, Z., Tie, Y., Olubiyi, O., Rigolo, L., Mehrtash, A., Norton, I., et al. (2015). Reconstruction of the arcuate fasciculus for surgical planning in the setting of peritumoral edema using two-tensor unscented Kalman filter tractography. NeuroImage Clin. 7, 815-822. doi: 10.1016/j.nicl.2015.03.009

Chen, Z., Tie, Y., Olubiyi, O., Zhang, F., Mehrtash, A., Rigolo, L., et al. (2016). Corticospinal tract modeling for neurosurgical planning by tracking through regions of peritumoral edema and crossing fibers using two-tensor unscented Kalman filter tractography. Int. J. Comput. Assist. Radiol. Surg. 11, 1475-1486. doi: $10.1007 /$ s11548-015-1344-5 generated. As XST works with the conventional tensorbased map, neurosurgical teams can easily incorporate this approach into their current analysis pipeline for reliable tract generation in a clinical environment. We would recommend that clinicians continue with their clinically approved SDT analysis but supplement it with these more advanced reconstruction methods for more accurate $\mathrm{CN}$ visualization.

\section{ETHICS STATEMENT}

This study was carried out in accordance with the recommendations of University Health Network (UHN) Research Ethics Board with written informed consent from all subjects. All subjects gave written informed consent in accordance with the Declaration of Helsinki. The protocol was approved by the University Health Network (UHN) Research Ethics Board.

\section{AUTHOR CONTRIBUTION}

$\mathrm{BB}$ and $\mathrm{MH}$ : Assisted with the design and conception of this work. BB, DD, and $\mathrm{MH}$ : Assisted with the acquisition of data for this work. BB, DC, and $\mathrm{MH}$ : Assisted with the data analysis for this work. BB, FS, EW, and MH: Assisted with the interpretation of data for this work. All authors were involved in the drafting of this work and giving final approval before submission. All authors agree to be accountable for all aspects of this work.

\section{FUNDING}

Elekta and Mitacs-Accelerate Canada IT02191.

Conturo, T. E., Lori, N. F., Cull, T. S., Akbudak, E., Snyder, A. Z., Shimony, J. S., et al. (1999). Tracking neuronal fiber pathways in the living human brain. Proc. Natl. Acad. Sci. U.S.A. 96, 10422-10427. doi: 10.1073/pnas.96.18.10422

Essayed, W. I., Zhang, F., Unadkat, P., Cosgrove, G. R., Golby, A. J., and O’Donnell, L. J. (2017). White matter tractography for neurosurgical planning: a tractography-based review of the current state of the art. Neuroimage Clin. 15, 659-672. doi: 10.1016/j.nicl.2017.06.011

Farquharson, S., Tournier, J. D., Calamante, F., Fabinyi, G., SchneiderKolsky, M., Jackson, G. D., et al. (2013). White matter fiber tractography: why we need to move beyond DTI. J. Neurosurg. 118, 1367-1377. doi: 10.3171/2013.2.JNS121294

Fedorov, A., Beichel, R., Kalpathy-Cramer, J., Finet, J., Fillion-Robin, J.-C., Pujol, S., et al. (2012). 3D slicer as an image computing platform for the quantitative imaging network. Magn. Reson. Imaging 30, 1323-1341. doi: 10.1016/j.mri.2012.05.001

Gerganov, V. M., Giordano, M., Samii, M., and Samii, A. (2011). Diffusion tensor imaging-based fiber tracking for prediction of the position of the facial nerve in relation to large vestibular schwannomas. J. Neurosurg. 115, 1087-1093. doi: 10.3171/2011.7.JNS11495

Hilly, O., Chen, J. M., Birch, J., Hwang, E., Lin, V. Y., Aviv, R. I., et al. (2016). Diffusion tensor imaging tractography of the facial nerve in patients with cerebellopontine angle tumors. Otol. Neurotol. 37, 388-393. doi: 10.1097/MAO.0000000000000984

Hodaie, M., Chen, D. Q., Quan, J., and Lapperiere, N. (2012). Tractography delineates microstructural changes in the trigeminal nerve after 
focal radiosurgery for trigeminal neuralgia. PLoS ONE 7:e32745. doi: 10.1371/journal.pone.0032745

Hodaie, M., Quan, J., and Chen, D. Q. (2010). In vivo visualization of cranial nerve pathways in humans using diffusion-based tractography. Neurosurgery 66, 788-795. discussion: 795-796. doi: 10.1227/01.NEU.0000367613.09324

Jenkinson, M., Beckmann, C. F., Behrens, T. E., Woolrich, M. W., and Smith, S. M. (2012). FSL. Neuroimage 62, 782-790. doi: 10.1016/j.neuroimage.2011.09.015

Jenkinson, M., Bannister, P. R., Brady, J. M., and Smith, S. M. (2002). Improved optimization for the robust and accurate linear registration and motion correction of brain images. Neuroimage 17, 825-841. doi: 10.1006/nimg.2002.1132

Jeurissen, B., Leemans, A., Tournier, J. D., Jones, D. K., and Sijbers, J. (2013). Investigating the prevalence of complex fiber configurations in white matter tissue with diffusion magnetic resonance imaging. Hum. Brain Mapp. 34, 2747-2766. doi: 10.1002/hbm.22099

Jones, D. K., Knosche, T. R., and Turner, R. (2013). White matter integrity, fiber count, and other fallacies: the do's and don'ts of diffusion MRI. Neuroimage 73, 239-254. doi: 10.1016/j.neuroimage.2012.06.081

Kabasawa, H., Masutani, Y., Aoki, S., Abe, O., Masumoto, T., Hayashi, N., et al. (2007). 3T PROPELLER diffusion tensor fiber tractography: a feasibility study for cranial nerve fiber tracking. Radiat. Med. 25, 462-466. doi: 10.1007/s11604-007-0169-8

Kuhnt, D., Bauer, M. H., Egger, J., Richter, M., Kapur, T., Sommer, J., et al. (2013a). Fiber tractography based on diffusion tensor imaging compared with high-angular-resolution diffusion imaging with compressed sensing: initial experience. Neurosurgery 72, 165-175. doi: 10.1227/NEU.0b013e318270d9fb

Kuhnt, D., Bauer, M. H., Sommer, J., Merhof, D., and Nimsky, C. (2013b). Optic radiation fiber tractography in glioma patients based on high angular resolution diffusion imaging with compressed sensing compared with diffusion tensor imaging - initial experience. PLOS ONE 8:e70973. doi: 10.1371/journal.pone.0070973

Massager, N., Lorenzoni, J., Devriendt, D., Desmedt, F., Brotchi, J., and Levivier, M. (2004). Gamma knife surgery for idiopathic trigeminal neuralgia performed using a far-anterior cisternal target and a high dose of radiation. J. Neurosurg. 100, 597-605. doi: 10.3171/jns.2004.100.4.0597

Mori, S., and Barker, P. B. (1999). Diffusion magnetic resonance imaging: its principle and applications. Anat. Rec. 257, 102-109. doi: 10.1002/(SICI)10970185(19990615)257:3<102::AID-AR7>3.0.CO;2-6

Mori, S., Crain, B. J., Chacko, V. P., and Van Zijk, P. C. (1999). Three-dimensional tracking of axonal projections in the brain by magnetic resonance imaging. Ann. Neurol. 45, 265-269. doi: 10.1002/1531-8249(199902)45:2<265::AIDANA21 $>3.0 . \mathrm{CO} ; 2-3$

Mori, S., and van Zijl, P. C. (2002). Fiber tracking: principles and strategies - a technical review. NMR Biomed. 15, 468-480. doi: 10.1002/nbm.781

Nimsky, C. (2014). Fiber tracking-we should move beyond diffusion tensor imaging. World Neurosurg. 82, 35-36. doi: 10.1016/j.wneu.2013.08.035

Nimsky, C., Bauer, M., and Carl, B. (2016). Merits and limits of tractography techniques for the uninitiated. Adv. Tech. Stand. Neurosurg. 43, 37-60. doi: 10.1007/978-3-319-21359-0_2

O'Donnell, L. J., and Westin, C. F. (2011). An introduction to diffusion tensor image analysis. Neurosurg. Clin. N. Am. 22, 185-196, viii. doi: 10.1016/j.nec.2010.12.004

Palesi, F., Tournier, J. D., Calamante, F., Muhlert, N., Castellazzi, G., Chard, D., et al. (2015). Contralateral cerebello-thalamo-cortical pathways with prominent involvement of associative areas in humans in vivo. Brain Struct. Funct. 6, 3369-3384. doi: 10.1007/s00429-014-0861-2

Potgieser, A. R., Wagemakers, M., Van Hulzen, A. L., De Jong, B. M., Hoving, E. W., and Groen, R. J. (2014). The role of diffusion tensor imaging in brain tumor surgery: a review of the literature. Clin. Neurol. Neurosurg. 124, 51-58. doi: 10.1016/j.clineuro.2014.06.009

Qazi, A. A., Radmanesh, A., O’Donnell, L., Kindlmann, G., Peled, S., Whalen, S., et al. (2009). Resolving crossings in the corticospinal tract by two- tensor streamline tractography: method and clinical assessment using fMRI. Neuroimage 47(Suppl. 2), T98-T106. doi: 10.1016/j.neuroimage.2008.06.034

Regis, J., Pellet, W., Delsanti, C., Dufour, H., Roche, P. H., Thomassin, J. M., et al. (2002). Functional outcome after gamma knife surgery or microsurgery for vestibular schwannomas. J. Neurosurg. 97, 1091-1100. doi: 10.3171/jns.2002.97.5.1091

Roundy, N., Delashaw, J. B., and Cetas, J. S. (2012). Preoperative identification of the facial nerve in patients with large cerebellopontine angle tumors using high-density diffusion tensor imaging. J. Neurosurg. 116, 697-702. doi: 10.3171/2011.12.JNS111404

Sammartino, D., King, N. K. K., Lozano, A. M., Schwartz, M. L., Huang, Y., and Hodaie, M. (2016). Tractography-based ventral intermediate nucleus targeting novel methodology and intraoperative validation. Mov. Disord. 31, 1217-1225. doi: $10.1002 / \mathrm{mds} .26633$

Soares, J. M., Marques, P., Alves, V., and Sousa, N. (2013). A hitchhiker's guide to diffusion tensor imaging. Front. Neurosci. 7:31. doi: 10.3389/fnins.2013. 00031

Tournier, J. D., Calamante, F., and Connelly, A. (2007). Robust determination of the fibre orientation distribution in diffusion MRI: non-negativity constrained super-resolved spherical deconvolution. Neuroimage 35, 1459-1472. doi: 10.1016/j.neuroimage.2007.02.016

Tournier, J. D., Calamante, F., and Connelly, A. (2012). MRtrix: diffusion tractography in crossing fiber regions. Int. J. Imaging Syst. Technol. 22, 53-66. doi: 10.1002/ima.22005

Tournier, J. D., Calamante, F., Gadian, D. G., and Connelly, A. (2004). Direct estimation of the fiber orientation density function from diffusionweighted MRI data using spherical deconvolution. Neuroimage 23, 1176-1185. doi: 10.1016/j.neuroimage.2004.07.037

Tournier, J. D., Mori, S., and Leemans, A. (2011). Diffusion tensor imaging and beyond. Magn. Reson. Med. 65, 1532-1556. doi: 10.1002/mrm.22924

Tuch, D. S., Reese, T. G., Wiegell, M. R., Makris, N., Belliveau, J. W., and Wedeen, V. J. (2002). High angular resolution diffusion imaging reveals intravoxel white matter fiber heterogeneity. Magn. Reson. Med. 48, 577-582. doi: $10.1002 / \mathrm{mrm} .10268$

Wedeen, V. J., Wang, R. P., Schmahmann, J. D., Benner, T., Tseng, W. Y., Dai, G., et al. (2008). Diffusion spectrum magnetic resonance imaging (DSI) tractography of crossing fibers. Neuroimage 41, 1267-1277. doi: 10.1016/j.neuroimage.2008.03.036

Westin, C. F., Maier, S. E., Mamata, H., Nabavi, A., Jolesz, F. A., and Kikinis, R. (2002). Processing and visualization for diffusion tensor MRI. Med. Image Anal. 6, 93-108. doi: 10.1016/S1361-8415(02)00053-1

Wilkins, B., Lee, N., Gajawelli, N., Law, M., and Lepore, N. (2015). Fiber estimation and tractography in diffusion MRI: development of simulated brain images and comparison of multi-fiber analysis methods at clinical b-values. Neuroimage 109, 341-356. doi: 10.1016/j.neuroimage.2014. 12.060

Yoshino, M., Kin, T., Ito, A., Saito, T., Nakagawa, D., Kamada, K., et al. (2015). Diffusion tensor tractography of normal facial and vestibulocochlear nerves. Int. J. Comput. Assist. Radiol. Surg. 10, 383-392. doi: $10.1007 / \mathrm{s} 11548-014-1129-2$

Conflict of Interest Statement: The authors declare that the research was conducted in the absence of any commercial or financial relationships that could be construed as a potential conflict of interest.

Copyright (c) 2017 Behan, Chen, Sammartino, DeSouza, Wharton-Shukster and Hodaie. This is an open-access article distributed under the terms of the Creative Commons Attribution License (CC BY). The use, distribution or reproduction in other forums is permitted, provided the original author(s) or licensor are credited and that the original publication in this journal is cited, in accordance with accepted academic practice. No use, distribution or reproduction is permitted which does not comply with these terms. 